\title{
Editorial
}

\section{Taking problem structuring methods forward}

\author{
Journal of the Operational Research Society (2007) 58, 545-546. \\ doi:10.1057/palgrave.jors.2602366
}

The articles in this issue of the Journal of the Operational Research Society complete the set of two special issues on problem structuring methods (Vol. 57, No. 7 and Vol. 58, No. 5). Since the first issue, a new Special Interest Group in Problem Structuring Methods sponsored by the Operational Research Society has been launched, well-attended PSM streams have been organized at the last OR and EURO conferences, and plans to organize an international PSM workshop are under way. All these activities suggest that the field continues to be 'alive and kicking'.

As with the first special issue, this second issue also contains articles that offer a variety of theoretical and empirical perspectives on the content and process of problem structuring methods. Both issues collectively provide insight into how current PSM research and practice is being shaped by the emerging generation of researchers and practitioners.

Part 1 of this issue presents new developments in problem structuring. Morton $e t$ al report on three case studies that used a distributed (ie different time, different place) SODA process. They explore similar work from the Delphi Policy and GSS literature, but contrast this with the distinguishing features of the action-oriented agenda of PSMs. They draw lessons from the action research regarding the circumstances when distributed PSMs are appropriate, reflecting particularly on the different types of task and the types of groups when they might be deployed. The following paper by O'Brien and Meadows takes the reader on a journey through the design and development of a new methodology which focuses on visioning. In supporting groups with their identification and consideration of alternative visions, Visioning Choices aims to assist organizations which seek a vision to which its stakeholders are willing to agree and work towards. Building collective understanding, harnessing creativity, structured process, involving multiple stakeholders, and modelling perceptions are all features of Visioning Choices. The authors argue that Visioning Choices sits comfortably alongside more established PSMs and, thus, should be regarded as a new PSM. In the last paper of this section, Bell and Morse reflect on their experiences of developing and applying a particular problem structuring method (Imagine) within contexts where issues of sustainability, environment, democracy, and conflict are critical. Based on these experiences, they go on to develop a sophisticated evaluation framework for deconstructing PSMbased projects in this kind of context.

Part 2 contains extensions and reflections on problem structuring. Horlick-Jones and Rosenhead trace the implicit use of empirical observation and tacit learning in historical applications of OR and explore the role that ethnographic methods could play in assisting the OR practitioner to make sense of a problem situation. They provide two PSM case studies which explicitly use ethnographic tools to provide a broader perspective of the problem's context; assist the targeting and deployment of the PSMs; and enable them to intervene with more authority. The paper also discusses the role of triangulating the outcomes of different methods and the practicality of cross disciplinary methodological hybridisation, but focuses on the practical advantages that such tools can bring to the OR practitioner. The next paper by Bryant provides an accessible, introductory account to and review of Drama Theory. Surprisingly, this will be the first publication of a full paper on Drama Theory in JORS and will hopefully bring the approach to the attention of a wider audience. The paper begins with a discussion of the theory's emergence, its development and use, while concluding with an agenda for future research directions in Drama Theory. The paper tackles the important issue for PSMs of ensuring their widespread adoption by offering some explanations as to why the uptake of Drama Theory has not been more prolific. Together with the issue of transferability, this has been a common theme across the two special issues, particularly the viewpoints. The section concludes with a paper by Papamichail et al, who conducted a unique study of the practice of facilitation. Four experienced facilitators worked with a similar 'client' group and each workshop (which included a significant problem structuring component) was video-taped. Their paper provides insight to: how the structure of the workshops differed across the facilitators; the facilitators' experiences (facilitators watched the videos and comment on their practice); the participants' experiences; the outcome from the workshops. We publish this paper to encourage reflective practitioners in all OR disciplines to think of new ways in which technology can further enhance the research process - in particular those who frequently engage clients in situations where process can be examined and improved.

Finally, Part 3 reports four novel applications. The first case by Wong provides a clear insight into how Robustness Analysis is applied in practice. After a brief review of Robustness Analysis, it then describes its development as a methodology to enhance its effectiveness in social contexts and its deployment in a participatory manner. The case itself is presented 
and reflected upon from a practitioner's perspective and involves a UK retailer developing its initial approach to online trading. The next two papers use system-based approaches as intervention tools. Foote et al provide a fascinating review of an intervention which used boundary critique and problem structuring during policy consultation. Boundary critique enabled the authors to understanding the interconnectivity and relevance of deep-rooted interests of a variety of community stakeholder groups. Rather than policy makers dismissing these interests as extraneous or irrelevant to the problem in hand, the analyses showed their genuine importance and impact which enabled re-framing of what was appropriate policy. Vo et al report on a success story of using Unbounded Systems Thinking (UST) within a Vietnamese organization suffering a steady decline in income for reasons unknown to those involved. Using the principles of UST and using causal mapping to structure their analyses, the researchers diagnosed the cause of the problem to be a significant gap between staff and managers' perspectives. Through modelling these gaps, they identified vicious loops which could have continued the decline. Management used the models to design action plans which broke the loops which helped to reverse the decline and staff used the models to better understand the legitimacy of managers' actions. Finally, the paper by Den-Hengst et al provides a detailed account of a collaborative simulation study within the Dutch Airline industry that builds explicitly upon what they called 'soft OR principles' and which include aspects such as outcome acceptance, stakeholder involvement and understanding through models. We publish this paper as part of this issue because we firmly believe that the feasibility of implementation in traditional hard OR projects can be significantly increased when adhered to these principles.

This issue concludes with a number of viewpoints and book reviews. Four viewpoints are offered by Mingers, Morrill,
Robinson and Vennix et al, who clearly articulate their particular perspectives about the present and the future of problem structuring methods as a discipline. The reviews include two books on systems-based modelling approaches, one on Dialogue Mapping and another one on facilitation.

We would like to draw your attention to another splendid paper on PSMs, one intended for the special issue but which appeared in the December 2006 issue of the journal. The paper by Checkland and Winters reflected on two uses of SSM in practice: one that focuses on the content of the perceived problematic situation of interest (SSMc), and the other devoted to the intellectual process of the intervention itself (SSMp). We regret that this paper does not appear with this collection of papers for it signifies an important development of, arguably, the most widely known PSM.

Our intention with these two special issues was to take stock of the PSM field and provide focus for the continued research and development of PSMs as a discipline beyond what has already been achieved by the originators of the methods. Needless to say, these issues do not represent a comprehensive treatment of PSMs. However, we hope they have moved us some distance towards understanding what possible directions are available for the future research and practice of PSMs.

Finally, as with the first issue, we would like again to express our sincere gratitude to all the authors and reviewers who have contributed to the production of this second issue, and to Sarah Parry and John Wilson for their patience and understanding throughout the whole process.
University of Warwick ${ }^{1}$

Aston University ${ }^{2}$

Attivation $^{3}$
Alberto Franco ${ }^{1}$
Duncan Shaw ${ }^{2}$
and

Mark Westcombe ${ }^{3}$ 